\title{
Role of the Lateral Preoptic Area in Sleep-Related Erectile Mechanisms and Sleep Generation in the Rat
}

\author{
Markus H. Schmidt, ${ }^{1}$ Jean-Louis Valatx, ${ }^{2}$ Kazuya Sakai, ${ }^{2}$ Patrice Fort, ${ }^{2}$ and Michel Jouvet $^{2}$ \\ ${ }^{1}$ Cleveland Clinic Foundation, Department of Neurology, Cleveland, Ohio 44195, and 2University of Claude Bernard, Institut \\ National de la Santé et de la Recherche Médicale U480, Lyon, France
}

\begin{abstract}
Penile erections are a characteristic phenomenon of paradoxical sleep (PS), or rapid eye movement sleep. Although the neural mechanisms of PS-related erections are unknown, the forebrain likely plays a critical role (Schmidt et al., 1999). The preoptic area is implicated in both sleep generation and copulatory mechanisms, suggesting it may be a primary candidate in PS erectile control. Continuous recordings of penile erections, body temperature, and sleep-wake states were performed before and up to 3 weeks after ibotenic acid lesions of the preoptic forebrain in three groups of rats. Neurotoxic lesions involving the medial preoptic area (MPOA) and anterior hypothalamus $(n=5)$ had no significant effects on either erectile activity or sleep-wake architecture. In contrast, bilateral lesions of the lateral preoptic region, with $(n=4)$ or without $(n=5)$ MPOA involvement, resulted in a significant decrease in the number of erections per hour of PS, number of PS-related erections, and PS phases exhibiting an erection. Lesion analysis revealed that the candidate structures
\end{abstract}

for PS erectile control include both the lateral preoptic area (LPOA) and ventral division of the bed nucleus of the stria terminalis; however, lesions of the LPOA were the most effective in disrupting PS erectile activity. LPOA lesioning also resulted in a long-lasting insomnia, characterized by the significant increase in wakefulness and decrease in slow wave sleep (SWS). PS architecture and waking-state erections remained unchanged after lesion in all groups. These data identify an essential role of the LPOA in both PS-related erectile mechanisms and SWS generation. Moreover, higher erectile mechanisms appear to be context-specific because LPOA lesioning selectively disrupted PS-related erections while leaving waking-state erections intact.

Key words: sleep-related erections; nocturnal penile tumescence; medial preoptic area; lateral preoptic area; bed nucleus of the stria terminalis; basal forebrain; insomnia; slow wave sleep; paradoxical sleep; REM sleep
Paradoxical sleep (PS), also known as rapid eye movement sleep, is characterized by several tonic and phasic phenomena, including cortical desynchronization, rapid eye movements, general muscle atonia, and penile erections. Whereas the neural control of PSrelated erections remains unknown, the executive mechanisms generating PS and its other tonic and phasic events are relatively well elucidated and localized within the mesopontine tegmentum and rostral medulla (Sakai 1985; Jones 1991).

Using a new technique of chronic penile erection recording in the rat (Schmidt et al., 1995), we recently demonstrated that mesencephalic transections disrupt PS erectile activity even though PS remains otherwise qualitatively intact (Schmidt et al., 1999). These data suggest that forebrain structures rostral to the transection are necessary for the production of PS-related erections. The source of this forebrain control currently is a matter of speculation.

Numerous forebrain structures have been implicated in reproductive mechanisms and, therefore, are potential candidates in sleep-related erectile control. Lesion, stimulation, and unit recording studies have elucidated an erectile role of the paraventricular nucleus (Melis et al., 1994), medial preoptic area (MPOA) (Giuliano et al., 1996), bed nucleus of the stria terminalis (BNST) (Valcourt and Sachs 1979), hippocampus (Chen et al., 1992), amygdala (Kondo 1992), and olfactory bulbs (Fernandez-Fewell and Meredith, 1995). Recent data suggest that some of these structures may be responsible for context-specific erectile control (Sachs, 1995).

We hypothesize that the preoptic area may be a primary fore-

\section{Received May 16, 2000; accepted May 31, 2000.}

This work was supported in part by Institut National de la Santé et de la Recherche Médicale U480, Claude Bernard University, Ohio Sleep Medicine Institute, Cleveland Clinic Foundation and the Medical College of Ohio at Toledo. We thank Dr. Helmut S. Schmidt, Dr. Pierre-Hervé Luppi, and Dr. Jian-Sheng Lin for their advice and critical review of this manuscript.

Correspondence should be addressed to Dr. Markus H. Schmidt, c/o Laura S. Tripepi, Department of Neurology, S90, Cleveland Clinic Foundation, 9500 Euclid Avenue, Cleveland, Ohio 44195. E-mail: schmidm@ccf.org.

Copyright (C) 2000 Society for Neuroscience $0270-6474 / 00 / 206640-08 \$ 15.00 / 0$ brain candidate in sleep-related erectile control given its role in both reproductive physiology and slow wave sleep (SWS) generation. The MPOA, for example, has long been implicated in copulatory mechanisms because its lesioning eliminates copulatory behavior in every species examined (Sachs and Meisel, 1988). Moreover, low androgen states disrupt PS-related erections in humans (Carani et al., 1992), and the MPOA contains androgensensitive neurons important for reproductive physiology (Cherry et al., 1992). With regard to sleep and wakefulness, both the MPOA and lateral preoptic area (LPOA) are described as "sleep centers" because their lesioning results in a long-lasting insomnia (Lucas and Sterman, 1975; Szymusiak, and McGinty 1986; Sallanon et al., 1989). Stimulation, neuroanatomical, and single-unit recording studies suggest an important role of the preoptic area in the generation of SWS and inhibition of waking mechanisms (Siegel and Wang, 1974; Ogawa and Kawamura, 1988; Sherin et al., 1998). Although the importance of the MPOA in copulatory behavior is well established, the relative contribution of the MPOA and LPOA in sleep generation remains controversial.

In the following experiments, cytotoxic lesions of the preoptic basal forebrain were performed using ibotenic acid in an attempt to localize forebrain structures involved in PS-related erections. Moreover, neurotoxic lesions primarily were limited to either the MPOA, LPOA, or both in three groups of rats to differentiate the relative contributions of these two structures in sleep-related erectile control and sleep generation. Continuous polygraphic recordings of penile erections, sleep-wake states, and body temperature were performed before and up to 3 weeks after neurotoxic lesions.

Preliminary results were presented in abstract form (Schmidt et al., 1996).

\section{MATERIALS AND METHODS}

Eighteen male Sprague Dawley rats (IFFA Credo, Arbresle, France) weighing $345-445 \mathrm{gm}$ body weight were used for these experiments. The rats were maintained on a $12 \mathrm{hr}$ light/dark cycle at an ambient temperature of $25.0 \pm 0.5^{\circ} \mathrm{C}$ with food and water available ad libitum throughout the experiment. 
Implantation. All rats were implanted for chronic penile erection monitoring and standard sleep recording under pentobarbital anesthesia $(60 \mathrm{mg} /$ $\mathrm{kg})$. Penile erections were recorded according to a technique previously described (Schmidt et al., 1994, 1995), involving chronic pressure monitoring within the bulb of the corpus spongiosum penis (CSP) and electromyography (EMG) of the bulbospongiosus (BS) muscles. Standard sleep recordings were performed in unanesthetized, freely behaving animals using dorsal neck EMG and cortical electroencephalography (EEG). A subcutaneous thermister was implanted in 12 rats for a continuous recording of body temperature. All electrical signals recorded from the rats were passed through an electronic swivel system (Air Precision) to allow free movement of the animals after implantation.

During the initial implantation, a 23 gauge stainless steel guide cannula was stereotaxically implanted bilaterally $3 \mathrm{~mm}$ above the target site within the preoptic area. A 26 gauge stainless steel stylet was placed inside the guide cannulae, protruding $1 \mathrm{~mm}$ beyond the guide cannula tip, to act as a protective plug. The coordinates of the guide cannulae were posterior $(\mathrm{P})$ $0.4-1.0 \mathrm{~mm}$ with respect to bregma, lateral (L) $0.5-1.3 \mathrm{~mm}$ with respect to the midline, and ventral (V) $4.0-5.2 \mathrm{~mm}$ with respect to the surface of the brain.

Cytotoxic lesions. After a 1 week postimplantation recovery period and two $24 \mathrm{hr}$ control recordings, the rats were anesthetized with ketamine $(80$ $\mathrm{mg} / \mathrm{kg}$ ). The protective stylets were removed from the guide cannulae, and $0.2-0.3 \mu \mathrm{l}$ of ibotenic acid $(45 \mu \mathrm{g} / \mu \mathrm{l})$ was injected bilaterally using a 26 gauge stainless steel or silicium (outer diameter, $150 \mu \mathrm{m}$; inner diameter, $75 \mu \mathrm{m}$ ) infusion cannula that protruded $3 \mathrm{~mm}$ beyond the guide cannula tip. Microinjections through the infusion cannula were performed at a rate of $0.02 \mu \mathrm{l} / \mathrm{min}$ using a $5.0 \mu \mathrm{l}$ Hamilton syringe connected to a microdrive pump. The infusion cannula was removed $10 \mathrm{~min}$ after the end of the injection, and the protective stylet was reinserted into the guide cannula. The animals were placed back into their home cages for postlesion recordings.

Data acquisition. Continuous polygraphic recordings were made before and after cytotoxic lesions with an ECEM polygraph. Continuous temperature recordings were performed on a personal computer (PC) at an acquisition rate of one data point every $30 \mathrm{sec}$. Each rat served as its own control with over 3 weeks of postlesion recordings. Wakefulness, SWS, and PS were scored from $30 \mathrm{sec}$ epochs using classical scoring criteria (Michel et al., 1961). Erectile events were scored in relation to CSP pressure changes. Briefly, an erectile event was defined as a minimum increase of 30 $\mathrm{mmHg}$ in CSP pressure with at least one pressure peak resulting from a BS muscle burst $>100 \mathrm{mmHg}$ above the "flaccid baseline" level. The end of the erectile event was defined as the moment when the CSP pressure fell below the $30 \mathrm{mmHg}$ threshold for $>15 \mathrm{sec}$. For each $24 \mathrm{hr}$ recording period, the following variables were then calculated: (1) the total number of erections during each behavioral state, (2) the number of erections per hour of each behavioral state, and (3) the percentage of PS phases associated with at least one erectile event

Histology. The rats were killed under deep pentobarbital anesthesia by transcardial perfusion of $250 \mathrm{ml}$ of Ringer's lactate solution $(0.1 \%$ heparin) followed by a $500 \mathrm{ml}$ of ice-cold solution of $4 \%$ paraformaldehyde and $0.2 \%$ picric acid in a $0.1 \mathrm{M}$ phosphate buffer. After a $24 \mathrm{hr}$ post-fixation in a solution of the same composition, the brains were transferred to a $0.1 \mathrm{M}$ PBS solution containing 30\% sucrose. The brains were later sliced in a cryostat into $25 \mu \mathrm{m}$ coronal sections. Free-floating sections were mounted on gelatin-coated slides.

Although demyelination has been described in proximity to the cannula injection site at the point of maximal gliosis in certain brain regions (Coffey et al., 1988), the hallmark of ibotenic acid cytotoxicity has long been considered its selective neuronal degeneration while leaving axons of passage intact (Schwarcz et al., 1979; Metzner and Juranek, 1997). Given its reliability in staining neuronal cell bodies, sections were stained with cresyl violet and analyzed by light microscopy to evaluate the extent of neuronal degeneration, the defining characteristic demarcating the neurotoxic lesion. Coronal sections were manually redrawn directly from mounted sections using the Biocom computer system and video-assisted microscopy.

Data analysis. Two $24 \mathrm{hr}$ control recordings, the fourth postlesion day, and two postlesion recordings from days 9 to 12 were used for data analysis. Paired $t$ tests and one-way ANOVAs of the means were used for statistical analyses. A post hoc Tukey's test was used for the ANOVA calculations on significant $(p<0.05) F$ values. All statistical analyses were performed with Statgraphics (Manugistics) software on a PC.

\section{RESULTS}

\section{Neurotoxic lesions}

Neurotoxic lesions were performed in a series of three groups involving a total of 18 rats. The groups differed only with respect to the location of the preoptic lesions. The medial preoptic, or MP, group $(n=5)$ exhibited similar bilateral lesions involving the MPOA while leaving the LPOA largely intact (Fig. 1). Lesions involved the anterior hypothalamic area $(\mathrm{AH})(n=5)$ and extended rostrally to the level of the anterior commissure $(n=3)$. In a second group of rats, larger cytotoxic lesions were undertaken to include both the MPOA and LPOA. This MP-LP group $(n=4)$ was noted by cytotoxic lesions of the MPOA with a distinct dorsolateral extension into the LPOA and ventral division of the BNST (Fig. 1). The ventrolateral preoptic nucleus (VLPO) was unilaterally lesioned in one rat from this group (Fig. 1, M12). Finally, the lateral preoptic, or LP, group $(n=5)$ involved lesions of the LPOA and ventral division of the BNST while leaving the MPOA intact (Fig. 1). Lesions in this group extended from the level of the $\mathrm{AH}$ through the ventral pallidum. The VLPO was unilaterally lesioned in only one rat (Fig. 1, U12), but otherwise was largely intact in all other cases.

Four of the 18 rats were not included in statistical analyses of sleep-wake or erection data. Two rats were found to have had only unilateral lesions, and an additional two rats were noted to have unique lesions likely secondary to errors in stereotaxic placement and could not be placed into one of the three groups. Although all rats were used for a final anatomical analysis and discussion, statistical analyses on sleep-wake and erection data were performed on the MP, MP-LP, and LP groups.

Cytotoxic lesions were characterized by the absence of neuronal cell bodies, replaced by a distinct glial formation without necrosis of neural tissue (Fig. 2). The suprachiasmatic nucleus (SCN), supraoptic nucleus (SO), and in more caudal lesions, the parvocellular and magnocellular divisions of the paraventricular nucleus (PVN) remained intact in all cases even though they were occasionally surrounded by cytotoxically lesioned neural tissue. Finally, the horizontal and vertical limbs of the diagonal bands (HDB) and magnocellular preoptic nuclei (MCPO) remained intact in all rats.

\section{Sleep-wake data}

Neurotoxic lesions in the MP group had no significant effects on sleep or wakefulness for any variable examined except for a significant short-term reduction in the total duration of SWS $\left(F_{(2,10)}=4.05, p<0.05\right)$ on day 4 after lesion (Fig. 3, Table 1$)$. This initial reduction in SWS was not associated with a significant change in the duration of PS or wakefulness on day 4. In addition, SWS duration recovered by day 10 after lesion.

Unlike the MP group, both the MP-LP (Table 2) and LP (Table 3) groups exhibited a marked insomnia involving a significant reduction in SWS (MP-LP: $F_{(2,8)}=10.98, p<0.01$; LP: $F_{(2,11)}=$ 42.56, $p<0.001)$ and a significant increase in total wakefulness $\left(\mathrm{MP}-\mathrm{LP}: F_{(2,8)}=9.45, p<0.01\right.$; LP: $\left.F_{(2,11)}=53.5, p<0.001\right)$ on both day 4 and circa day 10 after lesion (Fig. 3). This insomnia remained relatively unchanged from the circa day 10 level even after recording periods up to 3 weeks after lesion in rats from both groups.

Although the MP-LP and LP groups exhibited a long-term insomnia, only minor effects on PS were observed after preoptic lesions in these groups. For example, the total PS duration tended to decrease on day 4 after lesion in both groups (Fig. 3); however, this reduction did not reach statistical significance and it normalized by circa day 10 after lesion. In addition, the average, uninterrupted, PS episode duration decreased on only day 4 after lesion in the MP-LP group $\left(F_{(2,8)}=4.54 ; p<0.05\right)$, but also returned to normal by circa day 10 (Table 2 ). The average PS episode duration in the LP group, on the other hand, did not change significantly on either post-lesion days (Table 3), and the total number of PS episodes remained unchanged after lesion in all groups (Tables 2, 3).

\section{Erectile activity}

Penile erections were associated with an increase in CSP pressure from a flaccid baseline of $\sim 15-20 \mathrm{mmHg}$ to a tumescent pressure of $\sim 60-70 \mathrm{mmHg}$ (Fig. 4). As shown in Figure 4, erectile events during wakefulness and PS involved not only an increase in baseline erectile tissue pressure, but also dramatic, suprasystolic, CSP pressure peaks concurrent with BS muscle bursts. Erections during PS were associated with behavioral and physiological PS. The animals were curled in a sleeping posture with intermittent twitching of the whiskers and distal extremities, a muscle atonia was 


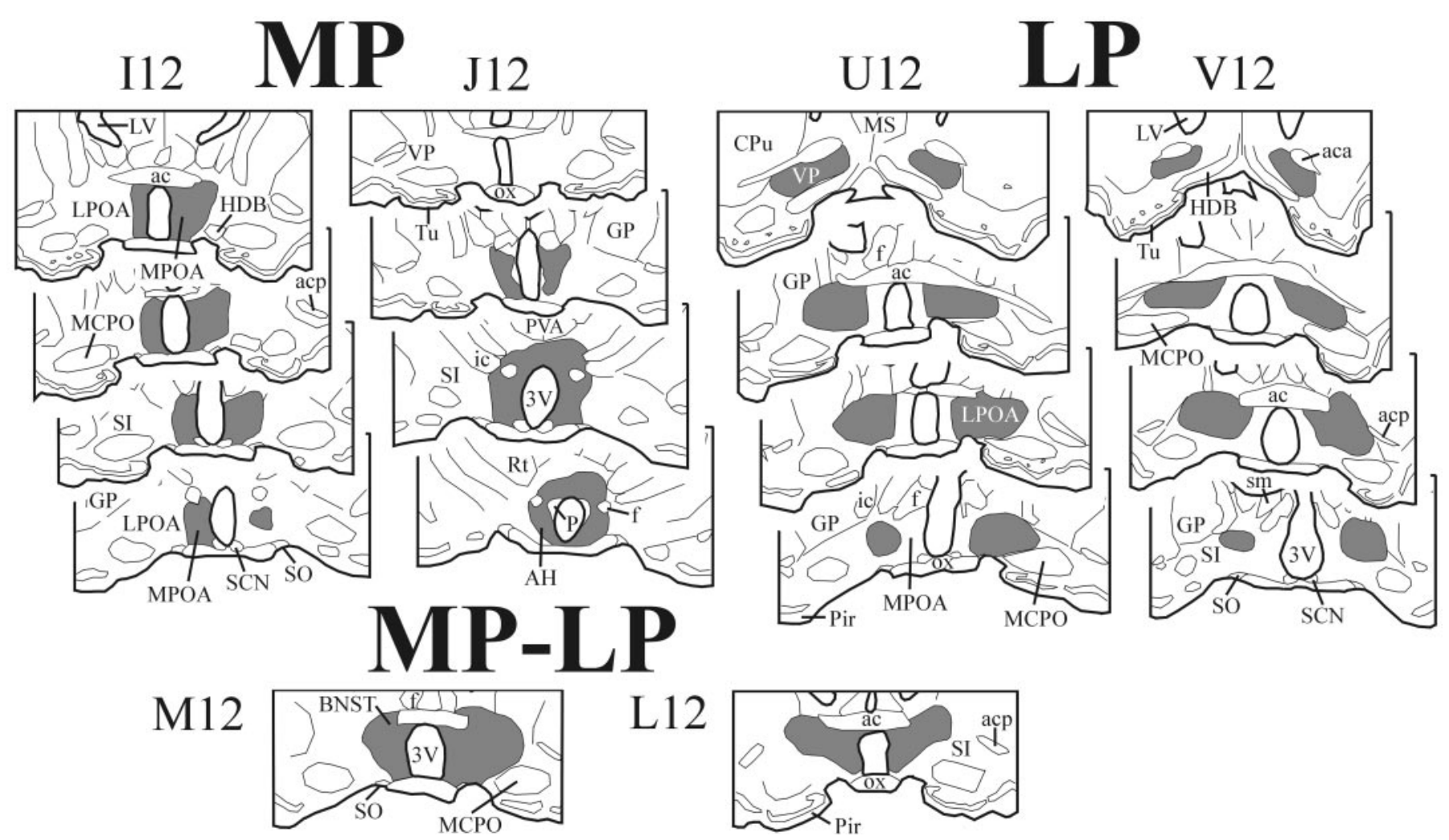

Figure 1. Cytotoxic lesions were performed in a series of three groups of rats that differed only with respect to the location of the lesions. This figure demonstrates a typical bilateral lesion (shaded area) of two rats from each of the three groups, i.e., the MP, MP-LP, and LP groups (see Results). $3 V$, Third ventricle; $a c$, anterior commissure; $a c a$, anterior commissure, anterior part; $a c p$, anterior commissure, posterior, posterior part; $A H$, anterior hypothalamic area; $B N S T$, bed nucleus of the stria terminalis; $C P u$, caudate putamen; $f$, fornix; $G P$, globus pallidus; $H D B$, nucleus of the horizontal limb of the diagonal band; ic, internal capsule; $L P O A$, lateral preoptic area; $L V$, lateral ventricle; $M C P O$, magnocellular preoptic nucleus; $M P O A$, medial preoptic area; $M S$, medial septal nucleus; $o x$, optic chiasm; $P$, hypothalamic paraventricular nucleus; Pir, piriform cortex; $P V A$, paraventricular thalamic nucleus; $R t$, reticular thalamic nucleus; $S C N$, suprachiasmatic nucleus; $S I$, substantia innominata; sm, stria medullaris of the thalamus; $S O$, supraoptic nucleus; $T u$, olfactory tubercle; $V P$, ventral pallidum.

observed in the neck EMG, and the EEG demonstrated a lowvoltage desynchronized pattern (Fig. 4). Erectile activity across sleep-wake states before cytotoxic lesions demonstrated a consistent relationship between the behavioral state of the animal and the probability of observing an erectile event. For example, rats from all three groups exhibited a mean of $\sim 10$ erections per hour of PS, four erections per hour of wakefulness, and a virtual absence of erections during SWS before lesioning (Fig. 5).

Sleep-related erectile activity remained unchanged after cytotoxic lesions in the MP group. We found no significant effects on either the number of erections per hour (Fig. 5), the total number of erections, or the percentage of PS phases exhibiting an erectile event (Table 1). Although PS erectile activity had a slight tendency to decrease after lesion in the MP group (Fig. 5), this decrease did not reach statistical significance.

PS-related erections in the MP-LP and LP groups, on the other hand, were severely disrupted by cytotoxic lesions (Fig. 4). As shown in Figure 5, the number of erections per hour of PS was significantly reduced after lesion in both groups (MP-LP: $F_{(2,8)}=$ 40.93, $p<0.001$; LP: $\left.F_{(2,11)}=25.48, p<0.001\right)$. This disruption in PS erectile activity was characterized by a total elimination of PS-related erections on day 4 in the MP-LP group and a decrease to $\sim 2$ erections per hour of PS on circa day 10 in both groups (Fig. 5). Similar reductions in PS-related erectile activity in these groups were observed with respect to the total number of erections during PS (MP-LP: $F_{(2,8)}=68.32, p<0.001 ; \mathrm{LP}: F_{(2,11)}=19.45, p<$ $0.001)$, as well as the percentage of PS phases exhibiting an erectile event (Tables 2, 3) (MP-LP: $F_{(2,8)}=69.94, p<0.001$; LP: $F_{(2,11)}$ $=17.60, p<0.001)$. Finally, this reduction in PS erectile activity in the MP-LP and LP groups remained at the circa day 10 level even after recording periods up to 3 weeks after lesion.
Unlike the disruptions to PS-related erections, waking-state erections were either unaffected or only moderately affected by preoptic lesions in all groups. For example, we found no effects on wakingstate erectile activity from cytotoxic lesions in either the MP or MP-LP groups, even though the latter group was characterized by a severe disruption in PS-related erections. The total number of erections during wakefulness (Tables 1,2), as well as the number of erections per hr of wakefulness (Fig. 5), remained unchanged after lesion in both groups. Although the number of erections per hour of wakefulness decreased significantly in the LP group after lesion (Fig. 5) $\left(F_{(2,10)}=30.56, p<0.001\right)$, this per hour reduction in waking-state erectile activity by circa day 10 appeared to be related primarily to the increase in wakefulness associated with the insomnia because the total number of erections during wakefulness at this time remained statistically unchanged in this group (Table 3 ). Finally, penile erections during wakefulness in all rats after lesion were qualitatively similar to prelesion controls (Fig. 4).

\section{Body temperature}

Twelve rats were implanted with a subcutaneous thermister for continuous body temperature recordings. All cytotoxic lesions of the preoptic area typically were associated with a short-term hypothermia in which the body temperature fell $\sim 2.0-3.0^{\circ} \mathrm{C}$ for the first 4-6 $\mathrm{hr}$ after the lesion. This hypothermia was followed by a long-lasting hyperthermia that was most apparent in the MP-LP group in which the body temperature increased an average of $1.6^{\circ} \mathrm{C}$ above control values for the first $3 \mathrm{~d}$ and only $1.0^{\circ} \mathrm{C}$ for the next $3 \mathrm{~d}$ after lesion. Moreover, all preoptic lesions were associated with increased circadian variations in body temperature. Body temperatures, however, generally returned to control values by day 10 after lesion in all rats. 

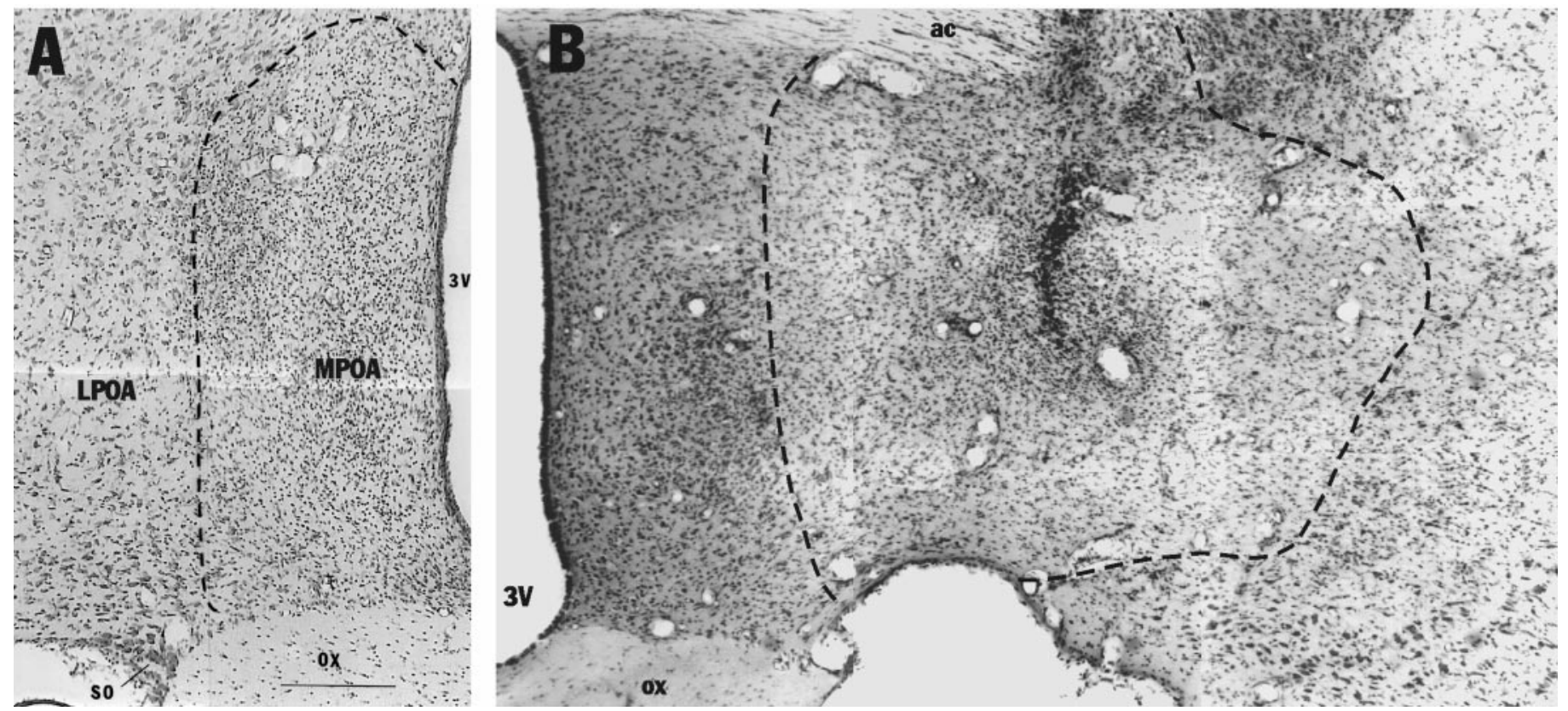

Figure 2. Photomicrographs of coronal sections through the preoptic area stained in cresyl violet demonstrating the typical quality of the neurotoxic lesions. Cytotoxic lesions were characterized by the absence of neuronal cell bodies, replaced by a distinct glial formation and without necrosis of neural tissue. The dashed lines represent the approximate extent of the lesions as defined by microscopic analysis. A, Cytotoxic lesion of the MPOA from rat I12 in Figure 1 of the MP group. Note that the LPOA remains intact in this rat from the MP group. $B$, Neurotoxic lesion of the LPOA from rat U12 in Figure 1 of the LP group demonstrating the center of the lesion as seen by the gliosis around the cannula tract. The photomicrograph shown in $B$ represents the LP lesion with the most ventral and medial extensions of the lesion. Abbreviations as in Figure 1.
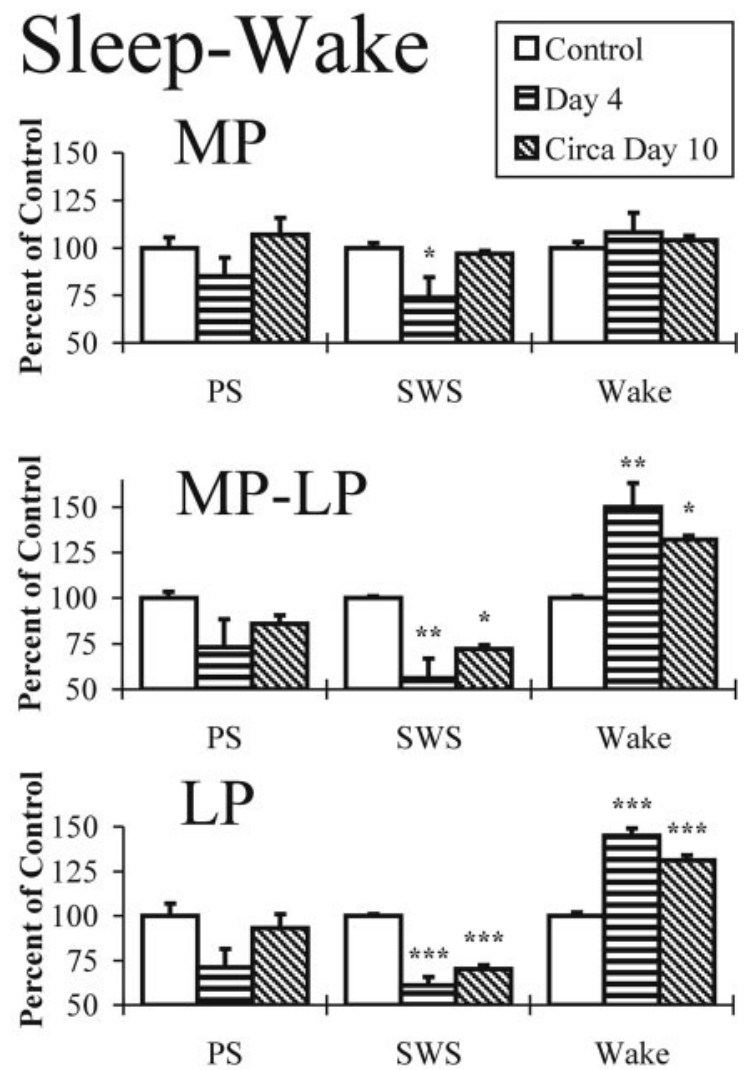

Figure 3. The total daily quantity of each behavioral state expressed as percentage of the control mean before (control) and after cytotoxic lesions on day 4 and circa day 10 in the MP, MP-LP, and LP groups. Asterisks indicate significant difference with respect to the corresponding prelesioned control using a one-way ANOVA and a post hoc Tukey test; ${ }^{*} p<0.05$, $* * p<0.01, * * * p<0.001$.

\section{Localization of an "effective zone" in PS erectile control}

Histological sections of all rats were analyzed individually as described and then reconstructed on common coronal planes corresponding to the atlas of Paxinos and Watson (1986). The lesioned areas that were bilaterally common for each rat were determined. As noted earlier, two rats were excluded from statistical analyses because of their unique bilateral lesions. One of these rats exhibited bilateral lesions restricted to rostral levels of the MPOA, as well as rostral ventromedial portions of the LPOA, near the level of the anterior commissure without affecting the caudal MPOA or the rostral $\mathrm{AH}$. The second rat had bilateral lesions involving the

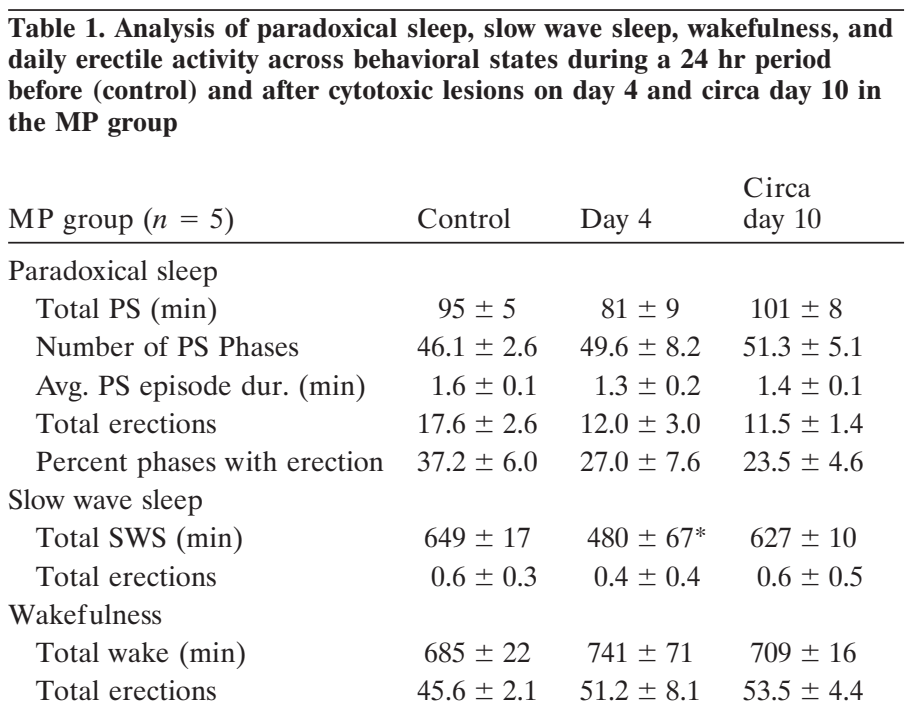

Values are given as the means \pm SEM. The data represent values from day 4 after lesion, as well as the mean values from two control recordings and two recordings obtained from the 9th to 12th day after lesion.

*Indicates a significant difference with respect to the corresponding prelesioned control using a one-way ANOVA and a post hoc Tukey test; * $p<0.05,{ }^{* *} p<0.01$, ${ }^{* * *} p<0.001$. 
Table 2. Analysis of paradoxical sleep, slow wave sleep, wakefulness, and daily erectile activity across behavioral states during a $24 \mathrm{hr}$ period before (control) and after cytotoxic lesions on day 4 and circa day 10 in the MP-LP group

\begin{tabular}{lrlc} 
MP-LP group $(n=4)$ & Control & Day 4 & $\begin{array}{l}\text { Circa } \\
\text { day } 10\end{array}$ \\
\hline Paradoxical sleep & & & \\
$\quad$ Total PS (min) & $90 \pm 3$ & $66 \pm 14$ & $77 \pm 4$ \\
$\quad$ Number of PS phases & $45.5 \pm 1.6$ & $46.0 \pm 8.6$ & $44.0 \pm 6.4$ \\
$\quad$ Avg. PS episode dur. (min) & $1.7 \pm 0.1$ & $1.0 \pm 0.2^{*}$ & $1.4 \pm 0.2$ \\
$\quad$ Total erections & $13.4 \pm 1.3$ & $0^{* * *}$ & $2.5 \pm 0.8^{* * *}$ \\
$\quad$ Percent phases with erection & $28.8 \pm 2.8$ & $0^{* * *}$ & $5.6 \pm 1.1^{* * *}$ \\
Slow wave sleep & & & \\
$\quad$ Total SWS (min) & $691 \pm 7$ & $386 \pm 75^{* *}$ & $496 \pm 17^{*}$ \\
$\quad$ Total erections & $0.6 \pm 0.2$ & 0 & $0.3 \pm 0.3$ \\
Wakefulness & & & \\
$\quad$ Total wake (min) & $659 \pm 7$ & $988 \pm 87^{* *}$ & $867 \pm 14^{*}$ \\
$\quad$ Total erections & $50.3 \pm 6.8$ & $49.5 \pm 2.0$ & $53.7 \pm 1.6$ \\
\hline
\end{tabular}

Values are given as the means \pm SEM. The data represent values from day 4 after lesion, as well as the mean values from two control recordings and two recordings obtained from the 9 th to 12 th day after lesion.

*Indicates a significant difference with respect to the corresponding prelesioned control using a one-way ANOVA and a post hoc Tukey test; ${ }^{*} p<0.05,{ }^{* *} p<0.01$, $* * * p<0.001$.

dorsomedial anterior hypothalamus and BNST rostrally, as well as ventromedial portions of the thalamus caudally. Both the MPOA and LPOA remained intact in this rat with the dorsal lesion. Because cytotoxic lesions in these two rats had no apparent effects on erectile activity, they were placed with the five rats from the MP group for this anatomical analysis. The anatomical results from these seven rats were combined, demarcating a zone encompassing the entire MPOA and medial anterior hypothalamus in which bilateral lesions had little or no effect on waking-state or PS erectile activity (Fig. 6, stippled area). A second zone was also determined which, when lesioned, resulted in a dramatic reduction in PSrelated erections. This "effective zone" included bilateral lesions that were common to all nine rats of the MP-LP and LP groups, but located outside of the noneffective zone. This "effective zone"

\begin{abstract}
Table 3. Analysis of paradoxical sleep, slow wave sleep, wakefulness, and daily erectile activity across behavioral states during a $24 \mathrm{hr}$ period before (control) and after cytotoxic lesions on day 4 and circa day 10 in the LP group
\end{abstract}

\begin{tabular}{lccc} 
LP group $(n=5)$ & Control & Day 4 & $\begin{array}{l}\text { Circa } \\
\text { day } 10\end{array}$ \\
\hline Paradoxical sleep & & & \\
$\quad$ Total PS (min) & $98 \pm 7$ & $70 \pm 10$ & $83 \pm 10$ \\
$\quad$ Number of PS Phases & $50.7 \pm 2.2$ & $43.4 \pm 5.3$ & $50.5 \pm 6.5$ \\
$\quad$ Avg. PS episode dur. (min) & $1.5 \pm 0.1$ & $1.1 \pm 0.2$ & $1.2 \pm 0.1$ \\
$\quad$ Total erections & $19.8 \pm 2.5$ & $3.2 \pm 1.4^{* * *}$ & $4.4 \pm 2.4^{* *}$ \\
$\quad$ Percent phases with erection & $36.8 \pm 3.7$ & $7.9 \pm 3.3^{* * *}$ & $9.5 \pm 5.1^{* *}$ \\
Slow wave sleep & & & \\
$\quad$ Total SWS (min) & $683 \pm 8$ & $419 \pm 32^{* * *}$ & $478 \pm 15^{* * *}$ \\
$\quad$ Total erections & $0.5 \pm 0.2$ & $0.6 \pm 0.2$ & $0.5 \pm 0.3$ \\
$\quad$ Wakefulness & & & \\
$\quad$ Total wake (min) & $656 \pm 13$ & $952 \pm 27^{* * *}$ & $859 \pm 20^{* * *}$ \\
$\quad$ Total erections & $61.2 \pm 3.1$ & $43.8 \pm 3.0^{* *}$ & $51.5 \pm 3.6$
\end{tabular}

Values are given as the means \pm SEM. The data represent values from day 4 after lesion, as well as the mean values from two control recordings and two recordings obtained from the 9th to 12th day after lesion.

*Indicates a significant difference with respect to the corresponding prelesioned control using a one-way ANOVA and a post hoc Tukey test; ${ }^{*} p<0.05$, ${ }^{* *} p<0.01$, *** $p<0.001$. was localized to the dorsomedial LPOA and the ventral division of the BNST near the anterior commissure (Fig. 6, blackened area).

\section{DISCUSSION}

Lesions of either the lateral preoptic region or MPOA have dramatically different effects on PS-related erections and SWS generation. Bilateral neurotoxic lesions of the lateral preoptic region resulted in a severe disruption in PS erectile activity while leaving PS architecture otherwise intact. These lateral lesions also produced a long-lasting insomnia involving a significant decrease in SWS and an increase in wakefulness. Similar lesions restricted to the MPOA, on the other hand, had little effect on either penile erections or sleep-wake architecture. Finally, the disruptive effects of lateral preoptic lesioning on erectile activity were specific to PS, leaving waking-state erections intact. These results identify an essential role of the lateral preoptic region in PS erectile neurophysiology and suggest a close anatomical association within this region of both PS-related erectile control and SWS-generating mechanisms.

\section{The preoptic area and erectile mechanisms}

Our data demonstrate that lesions of the lateral preoptic region, as in the MP-LP and LP groups, caused a significant decrease in the number of erections per hour of PS, total number of erections during PS, and percentage of PS phases exhibiting an erectile event with respect to prelesion controls. This disruption of PS-erectile activity could not be attributed to a disruption of PS architecture because the total daily quantity of PS and the average PS episode duration were minimally affected after lesion.

We hypothesize that the LPOA and ventral division of the BNST are the primary candidates in PS-erectile control because these structures encompassed the only lesioned areas bilaterally common to all rats in which PS-related erections were disrupted (Fig. 6). Although the relative contributions of these two structures remain to be clarified, rats with bilateral lesions encompassing both the ventral division of the BNST and LPOA were the most effective in disrupting PS-related erections, causing a complete elimination of such erections for the entire postlesion recording period. Similar lesions primarily involving the BNST while leaving the LPOA largely intact were less effective, resulting in an $\sim 60 \%$ reduction in PS-erectile activity.

Neuroanatomical (Moga et al., 1989; Westerhaus and Loewy, 1999), single-unit recording (Wilkinson and Pittman, 1995) and stimulation experiments (Inui et al., 1995) suggest that a subset of neurons within the LPOA and BNST may play an important role in hemodynamic regulation, at least in part through their connections with the hypothalamic PVN and vasomotor brainstem centers. The relationship between erectile mechanisms and vasomotor control within this lateral preoptic region remains to be explored. However, it is unlikely that our experimental observations are the result of a general perturbation in autonomic control because the disruptive effects of lateral preoptic lesioning on erectile activity were specific to PS while leaving waking-state erections intact.

This is the first study to our knowledge that implicates the LPOA in erectile mechanisms. Further investigation is required to elucidate its role in PS erectile control. Recent single-unit recording experiments demonstrate the presence of neurons within the LPOA which exhibit a specific unit firing activity during PS (Koyama and Hayaishi, 1994). It remains to be determined if these units may be specific to PS-related erections, i.e., "erection-on" neurons.

The BNST is a large structure with numerous subdivisions and it remains to be determined if the ventral division, which is implicated in PS erectile control in this study, is cytoarchitecturally related to that previously reported in reproductive mechanisms. Previous data suggest that the BNST may play a role in copulatory behavior (Emery and Sachs 1976; Valcourt and Sachs 1979), at least in part through the processing of olfactory cues. BNST lesions, for example, disrupt noncontact erections when in the vicinity of an estrous female, but leave copulatory erections relatively 


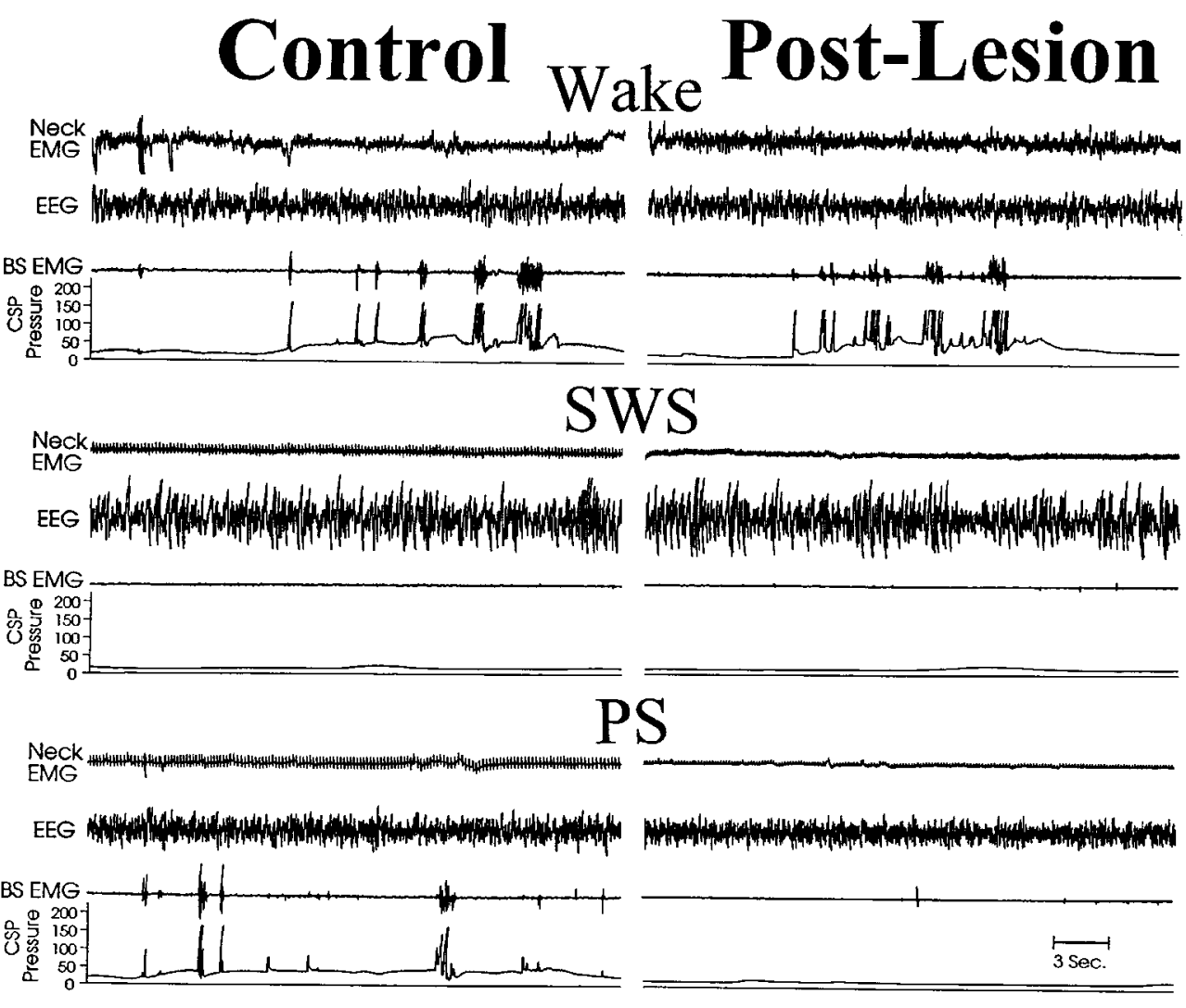

Figure 4. A typical polygraphic recording of wakefulness, SWS, and PS before (control) and circa day 10 after a bilateral cytotoxic lesion in a rat from the LP group. Control, Erectile events during wakefulness and PS were associated with an increase in baseline erectile tissue pressure and suprasystolic CSP pressure peaks concurrent with BS muscle bursts. SWS was noted by a general absence of penile erections. Post-Lesion, Wakingstate erections remained qualitatively and quantitatively intact after neurotoxic lesions, whereas PS-related erections were severely disrupted after lesion in this rat from the LP group.

intact once copulation is initiated (Liu et al., 1997). The BNST may transmit olfactory input related to copulatory behavior through its major reciprocal connections with the medial amygdala and MPOA (Liu et al., 1997).

Unlike the LPOA, for which little data are available concerning its role in reproductive mechanisms, the MPOA has long been implicated in reproductive physiology (Sachs and Meisel, 1988; Giuliano et al., 1995). Bilateral electrolytic (Ginton and Merari, 1977; Kondo and Arai, 1995) or neurotoxic (Hansen et al., 1982) lesions of the MPOA eliminate copulatory behavior, whereas stimulation of this area augments sexual activity (Merari and Ginton, 1975), generates penile erections (Giuliano et al., 1996), and removes a descending inhibition of penile reflexes (Marson and McKenna, 1994). Moreover, the unit activity of certain neurons in this structure increases in association with specific copulatory events (Shimura et al., 1994). Finally, c-fos expression in the MPOA increases after copulation in both male (Baum and Everett, 1992) and female (Erskine, 1993) rats. The MPOA is hypothesized to integrate and transmit copulation-related information arising from other forebrain structures, including the olfactory bulb, amygdala, and BNST (Sachs and Meisel, 1988), any of which when lesioned in isolation disrupt aspects of copulatory behavior.

Although the MPOA is essential for copulatory behavior, our data do not support a role of this structure in noncopulatory erectile control. We found that bilateral lesions encompassing the MPOA and anterior hypothalamus, such as in the MP group, had little or no effect on either waking-state or PS-related erectile activity. These findings are consistent with recent data demonstrating that males with MPOA lesions continue to exhibit noncontact erections when in the vicinity of receptive females even though copulatory behavior is severely compromised (Liu et al., 1997).

We found PS-related erections to be disrupted by lateral preoptic lesions even though waking-state erections remained relatively intact. This finding supports the hypothesis that erectile mechanisms of may be context-specific (Sachs, 1995; Liu et al., 1997). For example, penile erections can be reflexively elicited by tactile stimulation of the external genitalia without descending input from higher brain structures (Sachs and Meisel, 1988). With respect to supraspinal erectile control, stimulation of the hippocampus has been demonstrated to induce erectile events in rats (Chen et al., 1992), and one may speculate that the hippocampus and cortex may play a role in psychogenic erections generated from memory or fantasy. The olfactory bulbs, amygdala, and BNST, on the other hand, are preferentially involved in erections induced by olfactory cues (Sachs, 1995; Liu et al., 1997). Our data further support the hypothesis that context-specific erections, such as those generated during sleep, may involve specialized higher central mechanisms.

\section{The preoptic area and sleep generation}

The preoptic basal forebrain has long been considered a "sleep center" because of its role in sleep generation. Electrolytic or neurotoxic lesions of the preoptic area produce a long-lasting insomnia (McGinty and Sterman, 1968; Sallanon et al., 1989; Asala et al., 1990). In contrast, stimulation of this basal forebrain area produces cortical slow waves (Sterman and Clemente, 1962a; Siegel and Wang, 1974) and, in freely moving cats, induces SWS (Sterman and Clemente, 1962b). The preoptic area has been shown to contain neurons that are specifically active during SWS (SWS-on neurons) but are inactive during both wakefulness and PS (Ogawa and Kawamura, 1988; Szymusiak and McGinty, 1989).

Although the preoptic area is thought to play an active role in sleep generation, the relative contributions of the medial and lateral preoptic areas in this regard remain controversial. The second objective of these experiments, therefore, was to differentiate the relative contributions of these two regions in sleep-wake mechanisms.

Our data demonstrate that cytotoxic lesions encompassing the MPOA had no significant effects on sleep-wake architecture other than a transient short-term reduction in SWS duration without a corresponding increase in wakefulness. In contrast, bilateral lesions of the LPOA, leaving the MPOA intact, resulted in a long-lasting insomnia characterized by a significant decrease in SWS and an increase in wakefulness on both day 4 and circa day 10 post-lesion 


\section{Erections}
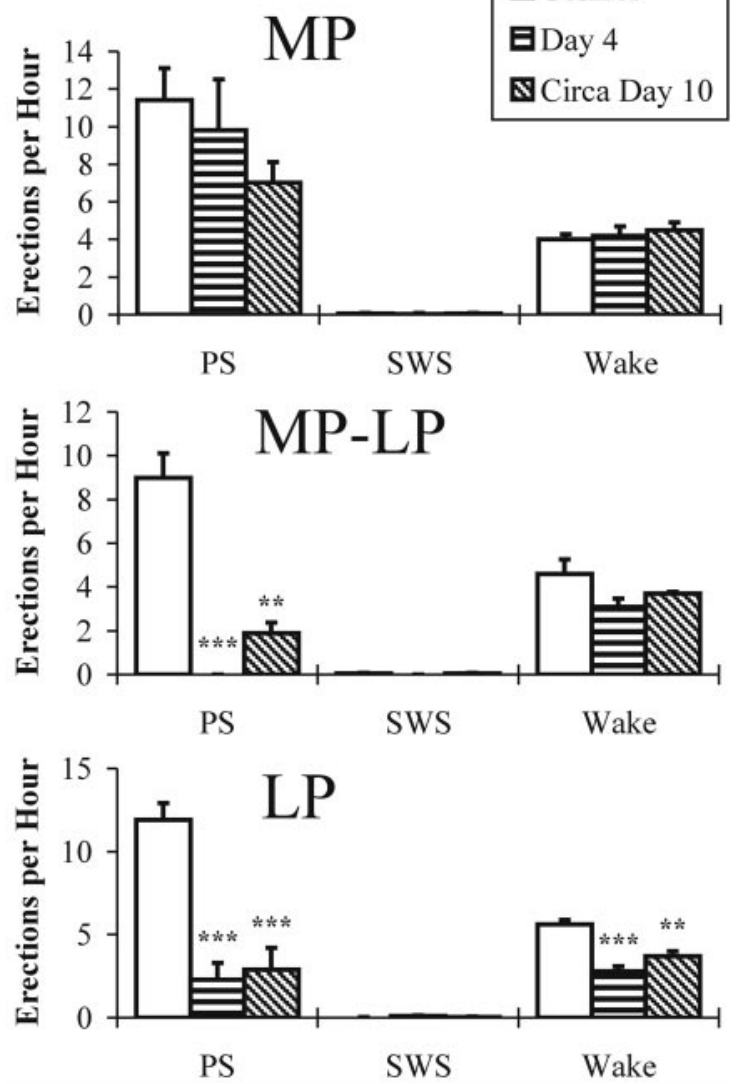

Figure 5. The number of erections per hour of each behavioral state before (control) and after cytotoxic lesions on day 4 and circa day 10 in the MP, MP-LP, and LP groups. Asterisks indicate significant difference with respect to the corresponding prelesioned control using a one-way ANOVA and a post hoc Tukey test; ${ }^{*} p<0.01,{ }^{* * *} p<0.001$.

(Fig. 3). These data clearly identify the LPOA, unlike the MPOA, as the preoptic region most important for sleep generation.

Recent data have implicated a specific cell group within the VLPO in SWS-generating mechanisms (Sherin et al., 1996). The immediate early gene c-fos is expressed by this cell group after long bouts of sleep, suggesting an active role in sleep generation (Sherin et al., 1996). Unit recording studies demonstrate an increase in the number of neurons exhibiting a specific unit firing activity during SWS as the recording electrode approaches the VLPO (Szymusiak et al., 1998). Finally, neuroanatomic data demonstrate a strong GABAergic projection from the VLPO to structures involved in maintaining wakefulness such as the histaminergic tuberomammillary nucleus (Sherin et al., 1998), the noradrenergic locus coeruleus (Luppi et al., 1998), and the cholinergic magnocellular preoptic nucleus (Fort et al., 1998). A recent in vitro study has further demonstrated that two-thirds of the VLPO neurons are GABAergic with characteristic electrophysiological properties and are uniformly inhibited by the major neurotransmitters involved in maintaining wakefulness, i.e., noradrenaline, serotonin, and acetylcholine (Gallopin et al., 2000). These data suggest that the VLPO may play a role in sleep generation through reciprocal inhibitory interactions with structures involved in wakefulness.

Although the VLPO may play a role in sleep generation, all rats in this study exhibited a long-lasting insomnia after LPOA lesions even though the VLPO remained intact in all but two cases. Moreover, we could find no correlation with respect to the ventral extent of the lesions and the degree of insomnia. Indeed, in the LP group, the rat with the most dorsal bilateral LPOA lesion exhibited more wakefulness after lesion than the rat in which the VLPO was

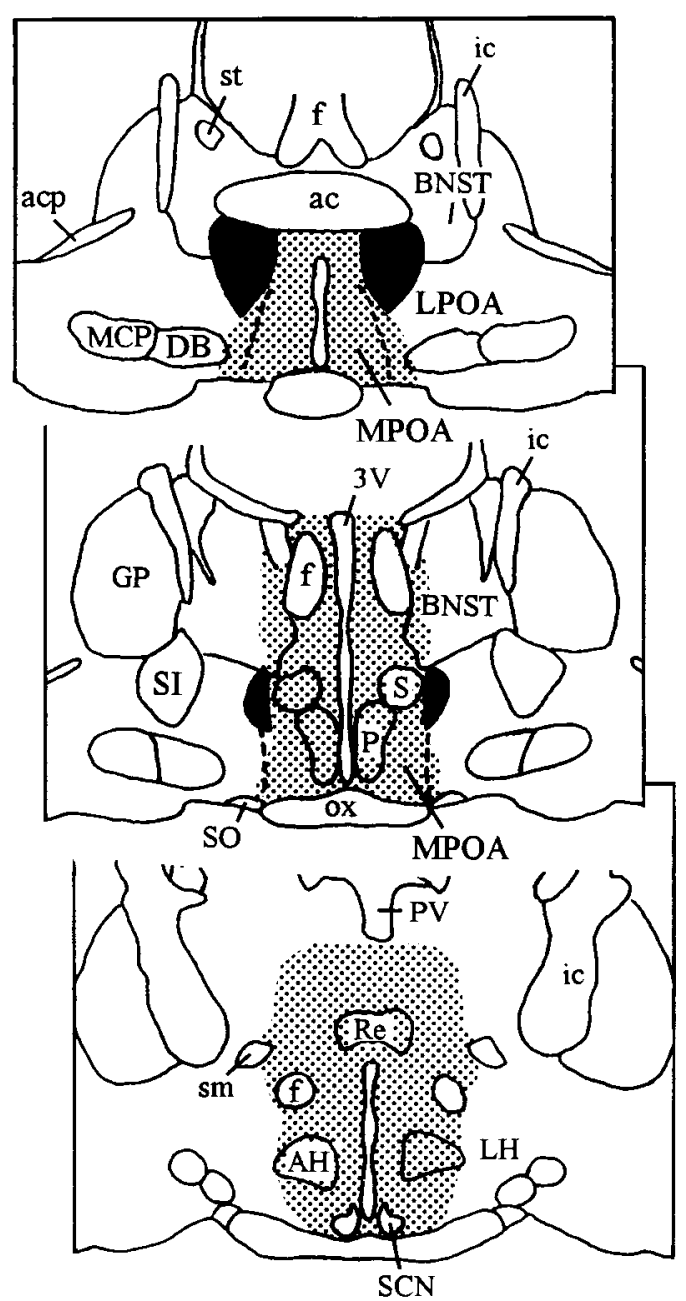

Figure 6. Summary of the effects of bilateral neurotoxic lesions on PSrelated penile erections. Blackened areas indicate an "effective zone" in which bilateral lesions dramatically reduced PS-related erections (common and symmetrical lesions obtained from nine rats in the MP-LP and LP groups), whereas bilateral lesions of the stippled areas had no effects on erectile activity. Abbreviations as in Figure 1.

lesioned. Our data suggest that neurons in the dorsal LPOA also play an important role in SWS generation.

\section{Summary}

These experiments suggest that the lateral preoptic region plays an essential role in both PS-related erections and SWS generation. The preoptic area encompasses a large group of heterogeneous neurons implicated in numerous physiological functions. Although these data suggest a close anatomic association within the lateral preoptic region of SWS mechanisms and PS erectile control, it remains to be determined if these neuronal populations are functionally related in this cytoarchitecturally diverse region.

The neural mechanisms of PS-related erections appear to be context-specific because the disruptive effects of lateral preoptic lesions on erectile activity were specific to PS while leaving wakingstate erections intact. The executive mechanisms of PS, as well as the subsystems that generate its tonic and phasic events, are located within the brainstem in the mesopontine tegmentum and rostral medulla (Jones, 1991). Although PS persists after mesencephalic transections or even after the complete removal of all neural elements rostral to the pons (Jouvet, 1967), PS-related erections are disrupted after lateral preoptic lesions. Further research is required to elucidate how the lateral preoptic region acts as a link between brainstem PS executive structures and descending spinal erectile control. 


\section{REFERENCES}

Asala SA, Okano Y, Honda K, Inoue S (1990) Effects of medial preoptic area lesions on sleep and wakefulness in unrestrained rats. Neurosci Lett 114:300-304.

Baum MJ, Everett BJ (1992) Increased expression of c-fos in the medial preoptic area after mating in male rats: role of afferent inputs from the medial amygdala and midbrain central tegmental field. Neuroscience 50:627-646.

Carani C, Bancroft J, Granata A, Del Rio G, Marrama P (1992) Testosterone and erectile function, nocturnal penile tumescence and rigidity, and erectile response to visual erotic stimuli in hypogonadal men. Psychopharmacology 17:647-654.

Chen K-K, Chan JYH, Chang LS, Chen M-T, Chan SHH (1992) Elicitation of penile erection following activation of the hippocampal formation in the rat. Neurosci Lett 141:218-222.

Cherry JA, Tobet SA, DeVoogd TJ, Baum MJ (1992) Effects of sex and androgen treatment on dendritic dimensions of neurons in the sexually dimorphic preoptic/anterior hypothalamic area of male and female ferrets. J Comp Neurol 323:577-585.

Coffey PJ, Perry VH, Allen Y, Sinden J, Rawlins JNP (1988) Ibotenic acid induced demyelination in the central nervous system: a consequence of a local inflammatory response. Neurosci Lett 84:178-184.

Emery DE, Sachs BD (1976) Copulatory behavior in male rats with lesions in the bed nucleus of the stria terminalis. Physiol Behav 17:803-806.

Erskine MS (1993) Mating-induced increases in FOS protein in preoptic area and medial amygdala of cycling female rats. Brain Res Bull 32:447-451.

Fernandez-Fewell GD, Meredith M (1995) Facilitation of mating behavior in male hamsters by LHRH and AcLHRH5-10: interaction with the vomeronasal system. Physiol Behav 57:213-221.

Fort P, Gervasoni D, Peyron C, Rampon C, Boissard R, Luppi PH (1998) GABAergic projections to the magnocellular preoptic area and substantia innominata in the rat. Soc Neurosci Abstr 24:1694.

Gallopin T, Fort P, Eggermann E, Cauli B, Luppi PH, Rossier J, Audinat E, Mühlethaler M, Serafin M (2000) Identification of sleep-promoting neurons in vitro. Nature 404:992-995.

Ginton A, Merari A (1977) Long range effects of MPOA lesion on mating behavior in the male rat. Brain Res 120:158-163.

Giuliano FA, Rampin O, Benoit G, Jardin A (1995) Neural control of penile erection. Urol Clin North Am 22:747-766.

Giuliano F, Rampin O, Brown K, Courtois F, Benoit G, Jardin A (1996) Stimulation of the medial preoptic area of the hypothalamus in the rat elicits increases in intracavernous pressure. Neurosci Lett 209:1-4.

Hansen S, Köhler C, Goldstein M, Steinbusch HVM (1982) Effects of ibotenic acid-induced neuronal degeneration in the medial preoptic area and the lateral hypothalamic area on sexual behavior in the male rat. Brain Res 239:213-232.

Inui K, Nomura J, Murase S, Nosaka S (1995) Facilitation of the arterial baroreflex by the preoptic area in anaesthetized rats. J Physiol (Lond) 488 2:521-531.

Jones BE (1991) Paradoxical sleep and its chemical/structural substrates in the brain. Neuroscience 40:637-656.

Jouvet M (1967) Neurophysiology of the states of sleep. Physiol Rev 47:117-177.

Kondo Y (1992) Lesions of the medial amygdala produce severe impairment of copulatory behavior in sexually inexperienced male rats. Physiol Behav 51:939-943.

Kondo Y, Arai Y (1995) Functional association between the medial amygdala and the medial preoptic area in regulation of mating behavior in the male rat. Physiol Behav 57:69-73.

Koyama Y, Hayaishi O (1994) Firing of neurons in the preoptic/anterior hypothalamic areas in rat: Its possible involvement in slow wave sleep and paradoxical sleep. Neurosci Res 19:31-38.

Liu YC, Salamone JD, Sachs BD (1997) Lesions in medial preoptic area and bed nucleus of stria terminalis: differential effects on copulatory behavior and noncontact erection in male rats. J Neurosci 17:5245-5253.

Lucas EA, Sterman MB (1975) Effects of a forebrain lesion on the polycyclic sleep-wake cycle and sleep-wake patterns in the cat. Exp Neurol 46:368-388.

Luppi PH, Peyron C, Rampon C, Gervasoni D, Barbagli B, Boissard R, Fort P (1998) Inhibitory mechanisms in the raphe dorsalis and locus coeruleus during sleep. In: Handbook of behavioral state control: cellular and molecular mechanisms (Lydic R, Baghdoyan H, eds), pp 195-211. Boca Raton, FL: CRC.

Marson L, McKenna KE (1994) Stimulation of the hypothalamus initiates the urthrogenital reflex in male rats. Brain Res 638:103-108.

McGinty DJ, Sterman MB (1968) Sleep suppression after basal forebrain lesions in the cat. Science 160:1253-1255.

Melis MR, Stancampiano R, Argiolas A (1994) Penile erection and yawn- ing induced by paraventricular NMDA injection in male rats are mediated by oxytocin. Pharmacol Biochem Behav 48:203-207.

Merari A, Ginton A (1975) Characteristics of exaggerated sexual behavior induced by electrical stimulation of the medial preoptic area in male rats. Brain Res 86:97-108.

Metzner W, Juranek J (1997) A method to biotinylate and histochemically visualize ibotenic acid for pharmacological inactivation studies. J Neurosci Methods 76:143-150

Michel F, Klein M, Jouvet D, Valatx JL, Jouvet M (1961) Etude polygraphic du sommeil chez le rat. C R Acad Sci 12:2389-2392.

Moga MM, Saper CB, Gray TS (1989) Bed nucleus of the stria terminalis: cytoarchitecture, immunohistochemistry, and projection to the parabrachial nucleus in the rat. J Comp Neurol 283:315-332.

Ogawa Y, Kawamura H (1988) Increase of multiple unit activity during slow wave sleep in the cat preoptic area. Brain Res Bull 20:897-902.

Paxinos G, Watson C (1986) The rat brain in stereotaxic coordinates. Tokyo: Academic.

Sachs BD (1995) Placing erection in context: the reflexogenic-psychogenic dichotomy reconsidered. Neurosci Biobehav Rev 19:211-224.

Sachs BD, Meisel RL (1988) The physiology of male sexual behavior. In: The Physiology of Reproduction (Knobil E, Neill J, eds), pp 1393-1485. New York: Raven.

Sakai K (1985) Anatomical and physiological basis of paradoxical sleep. In: Brain mechanisms of sleep (McGinty DJ, Drucker-Colin R, Morrison A, Parmeggiani, eds), pp 111-137. New York: Raven.

Sallanon M, Denoyer M, Kitahama K, Aubert C, Gay N, Jouvet M (1989) Long lasting insomnia induced by preoptic neuron lesions and its transient reversal by muscimol injection into the posterior hypothalamus in the cat. Neuroscience 32:669-683.

Schmidt MH, Valatx JL, Schmidt HS, Wauquier A, Jouvet M (1994) Experimental evidence of penile erections during paradoxical sleep in the rat. NeuroReport 5:561-564.

Schmidt MH, Valatx JL, Sakai K, Debilly G, Jouvet M (1995) Corpus spongiosum penis pressure and perineal muscle activity during reflexive erections in the rat. Am J Physiol 269:R904-R913.

Schmidt MH, Valatx JL, Sakai K, Jouvet M (1996) The basal forebrain and the control of sleep-related penile erections: evidence from cytotoxic lesions in the rat. Sleep Res 25:25.

Schmidt MH, Sakai K, Valatx JL, Jouvet M (1999) The effects of spinal or mesencephalic transections on sleep-related erections and ex-copula penile reflexes in the rat. Sleep 22:409-418.

Schwarcz R, Hokfelt T, Fuxe K, Jonsson G, Goldstein M, Terenius L (1979) Ibotenic acid-induced neuronal degeneration: a morphological and neurochemical study. Exp Brain Res 37:199-216.

Sherin JE, Elmquist JK, Torrealba F, Saper CB (1998) Innervation of histaminergic tuberomammillary neurons by GABAergic and Galaninergic neurons in the ventrolateral preoptic nucleus of the rat. J Neurosci 18:4705-4721.

Sherin JE, Shiromani PJ, McCarley RW, Saper CB (1996) Activation of ventrolateral preoptic neurons during sleep. Science 271:216-219.

Shimura T, Yamamoto T, Shimokochi M (1994) The medial preoptic area is involved in both sexual arousal and performance in male rats: reevaluation of neuron activity in freely moving animals. Brain Res 640:215-222.

Siegel J, Wang RY (1974) Electroencephalographic, behavioral, and single-unit effects produced by stimulation of forebrain inhibitory structures in cats. Exp Neurol 42:28-50.

Sterman MB, Clemente CD (1962a) Forebrain inhibitory mechanisms: cortical synchronization induced by basal forebrain stimulation. Exp Neurol 6:91-102.

Sterman MB, Clemente CD (1962b) Forebrain inhibitory mechanisms: Sleep patterns induced by basal forebrain stimulation in the behaving cat. Exp Neurol 6:103-117.

Szymusiak R, McGinty D (1986) Sleep suppression following kainic acidinduced lesions of the basal forebrain. Exp Neurol 94:598-614.

Szymusiak R, McGinty D (1989) Sleep-waking discharge of basal forebrain projection neurons in cats. Brain Res Bull 22:423-430.

Szymusiak R, Alam N, Steininger TL, McGinty D (1998) Sleep-waking discharge patterns of ventrolateral preoptic/anterior hypothalamic neurons in rats. Brain Res 803:178-188.

Westerhaus MJ, Loewy A (1999) Sympathetic-related neurons in the preoptic region of the rat identified by viral transneuronal labeling. J Comp Neurol 414:361-378

Wilkinson MF, Pittman QJ (1995) Changes in arterial blood pressure alter activity of electrophysiologically identified single units of the bed nucleus of the stria terminalis. Neuroscience 64:835-844.

Valcourt RJ, Sachs BD (1979) Penile reflexes and copulatory behavior in male rats following lesions in the bed nucleus of the stria terminalis. Brain Res Bull 4:131-133. 